\title{
Time Domain Synchronous OFDM Based on Simultaneous Multi-Channel Reconstruction
}

\author{
Linglong Dai, Jintao Wang and Zhaocheng Wang \\ Tsinghua National Laboratory for Information \\ Science and Technology, Tsinghua University, China \\ E-mail: daill@tsinghua.edu.cn
}

\author{
Paschalis Tsiaflakis, Marc Moonen \\ Department of Electrical Engineering (ESAT-SCD), \\ Katholieke Universiteit Leuven (KUL), Belgium \\ E-mail: marc.moonen@esat.kuleuven.be
}

\begin{abstract}
Time domain synchronous OFDM (TDS-OFDM) can achieve a higher spectrum efficiency than standard cyclic prefix OFDM (CP-OFDM). Currently, it can support constellations up to 64QAM, but cannot support higher-order constellations like 256QAM due to the residual mutual interferences between the pseudorandom noise (PN) guard interval and the OFDM data block. To solve this problem, we break the traditional approach of iterative interference cancellation and propose the idea of using multiple inter-block-interference (IBI)-free regions of very small size to realize simultaneous multi-channel reconstruction under the framework of structured compressive sensing, whereby the sparsity nature of wireless channels as well as the characteristic that path delays vary much slower than path gains are jointly exploited. In this way, the mutually conditional time-domain channel estimation and frequency-domain data demodulation in TDS-OFDM can be decoupled without the use of IBI removal. We then propose the adaptive simultaneous orthogonal matching pursuit (A-SOMP) algorithm with low complexity to realize accurate multi-channel reconstruction, whose performance is close to the Cramér-Rao lower bound (CRLB). Simulation results confirm that the proposed scheme can support 256QAM without changing the current signal structure, so the spectrum efficiency can be increased by about $30 \%$.
\end{abstract}

\section{INTRODUCTION}

OFDM has been widely recognized as a prominent physical layer technique for future wireless communications [1], [2]. There are basically three types of OFDM [3]: cyclic prefix OFDM (CP-OFDM), zero padding OFDM (ZP-OFDM), and time domain synchronous OFDM (TDS-OFDM). The most widely used CP-OFDM utilizes a $\mathrm{CP}$ as the guard interval in between successive OFDM data blocks to alleviate inter-blockinterference (IBI) [4]. The $\mathrm{CP}$ is replaced by a zero padding in ZP-OFDM [4]. Unlike CP-OFDM or ZP-OFDM, TDSOFDM adopts a known pseudorandom noise (PN) sequence as the guard interval as well as the training sequence (TS) for time-domain synchronization and channel estimation. Hence, a higher spectrum efficiency can be achieved due to the avoidance of frequency-domain pilots for channel estimation as adopted by CP-OFDM and ZP-OFDM [5]. TDS-OFDM is the key technology of the international digital television broadcasting standard called digital television/terrestrial multimedia broadcasting (DTMB), which bas been proposed by China, and has been successfully deployed in China, Cuba, Cambodia, etc [5].

However, the mutual interferences between the PN sequence and the OFDM data block in TDS-OFDM make time-domain channel estimation and frequency-domain data demodulation mutually conditional, so an iterative interference cancellation algorithm [6] has to be implemented, which unfortunately cannot remove the interferences completely. Due to this, it is difficult for TDS-OFDM to support interference-sensitive high-order constellations like 256QAM in multipath channels with large delay spread [5]. Currently, the highest modulation order that can be supported by TDS-OFDM is 64QAM, while $\mathrm{CP}-\mathrm{OFDM}$ in the recently announced next-generation digital television broadcasting standard called DVB-T2 [7] can support 256QAM to achieve higher spectrum efficiency. One attractive solution is the dual PN padding OFDM (DPNOFDM) scheme [8], whereby two repeated PN sequences are inserted in every TDS-OFDM symbol to avoid the interference from the OFDM data block into the second PN sequence. However, the extra PN sequence decreases the spectrum efficiency, especially when the original guard interval length is long such as in wireless broadcasting systems.

In this paper, without changing the current signal structure, we propose a TDS-OFDM transmission scheme based on simultaneous multi-path reconstruction. To enable this, two channel properties are jointly exploited, namely the channel sparsity and the fact that path delays vary much slower than path gains, which are usually not considered in conventional OFDM systems. We break the traditional approach where the interference imposed on the received PN sequence is cancelled, and propose the idea of using multiple IBI-free regions of very small size to realize simultaneous multi-channel reconstruction by utilizing the newly emerging theory of structured compressive sensing (CS) [9]. This mechanism requires no iterative interference cancellation to decouple the mutually conditional time-domain channel estimation and frequencydomain data detection. Furthermore, by jointly utilizing the classical signal recovery algorithm called simultaneous orthogonal matching pursuit (SOMP) [9] and the specific timefrequency features of TDS-OFDM, we propose the adaptive SOMP (A-SOMP) algorithm to realize simultaneous multichannel reconstruction with reduced complexity. In addition, we also provide the theoretical Cramér-Rao lower bound (CRLB) of the simultaneous multi-channel reconstruction method, which is closely approached as demonstrated by the simulation results.

The rest of this paper is organized as follows. Section II 


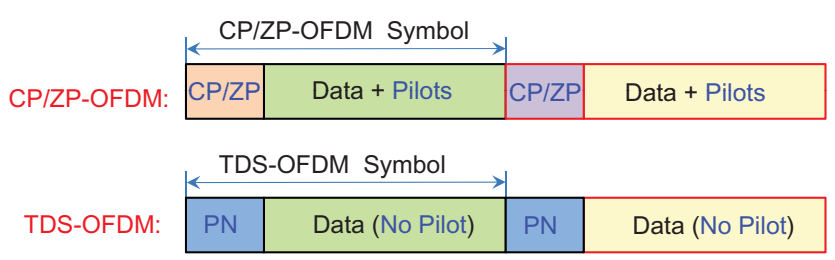

(a)

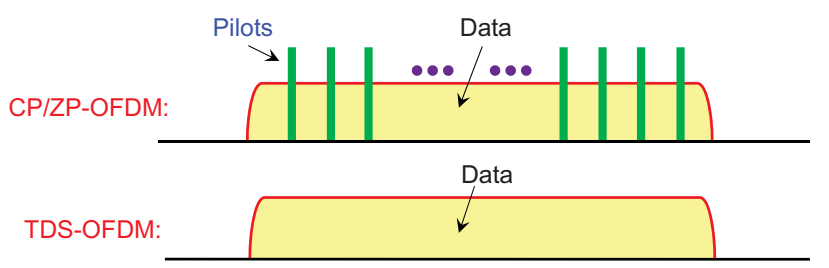

(b)

Fig. 1. Signal structure comparison between CP-OFDM, ZP-OFDM and TDS-OFDM: (a) in the time domain; (b) in the frequency domain.

presents the proposed TDS-OFDM scheme based on simultaneous multi-channel reconstruction. Then, the simultaneous multi-channel reconstruction method based on A-SOMP is proposed in Section III. In Section IV, simulation results are presented to investigate the performance of the proposed scheme. Finally, conclusions are drawn in Section V.

Notation: We use boldface letters to denote matrices and column vectors; 0 denotes the zero matrix; $\otimes$ denotes the circular correlation; $(\cdot)^{T},(\cdot)^{H},(\cdot)^{-1},(\cdot)^{\dagger}$, and $\|\cdot\|_{p}$ denote the transpose, conjugate transpose, matrix inversion, MoorePenrose matrix inversion, and $l_{p}$ norm operation, respectively; $\mathbf{x}_{r}$ is generated by restricting the vector $\mathbf{x}$ to its $r$ largest components; $\left.\mathbf{x}\right|_{\Gamma}$ denotes the entries of the vector $\mathbf{x}$ in the set $\Gamma ; \boldsymbol{\Phi}_{\Gamma}$ denotes the column submatrix comprising the $\Gamma$ columns of $\boldsymbol{\Phi} ; \Gamma^{c}$ is the complementary set of $\Gamma$.

\section{TDS-OFDM BASED ON Simultaneous Multi-ChanNel ReCONSTRUCTION}

Fig. 1 compares the signal structure of CP-OFDM, ZPOFDM and TDS-OFDM in the time and frequency domain. The $i$ th TDS-OFDM symbol $\mathbf{s}_{i}=\left[s_{i, 0}, s_{i, 1}, \cdots, s_{i, M+N-1}\right]^{T}$ comprises the time-domain known PN sequence $\mathbf{c}_{i}=$ $\left[c_{i, 0}, c_{i, 1}, \cdots, c_{i, M-1}\right]^{T}$ of length $M$ and the OFDM data block $\mathbf{x}_{i}=\left[x_{i, 0}, x_{i, 1}, \cdots, x_{i, N-1}\right]^{T}$ of length $N$, and it is denoted as

$$
\mathbf{s}_{i}=\left[\begin{array}{cc}
\mathbf{c}_{i}^{T} & \mathbf{x}_{i}^{T}
\end{array}\right]^{T} .
$$

Note that in contrast to CP-OFDM and ZP-OFDM, TDSOFDM has no pilots in the frequency domain.

As illustrated in Fig. 2, the mutual interferences between the PN sequences and the OFDM data blocks make channel estimation and data demodulation mutually conditional. Hence, an iterative interference cancellation has been proposed to refine channel estimation and data demodulation iteratively, but the complete removal of the residual interferences is difficult [6].

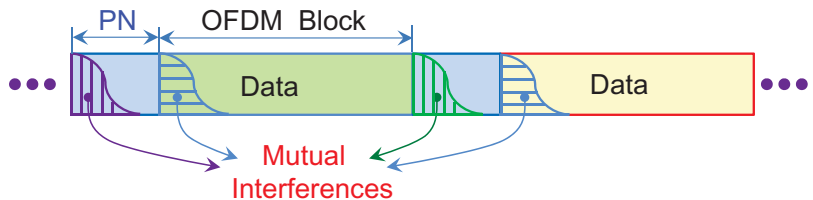

Fig. 2. The mutual interferences between the PN sequence and the OFDM data block in multipath channels.

Unlike the pilot-based frequency-domain channel estimation in CP-OFDM and ZP-OFDM [5], TDS-OFDM realizes timedomain channel estimation based on the received PN sequence $\mathbf{d}_{i}=\left[d_{i, 0}, d_{i, 1}, \cdots, d_{i, M-1}\right]^{T}$ denoted by

$$
\mathbf{d}_{i}=\boldsymbol{\Psi}_{i} \mathbf{h}_{i}+\mathbf{v}_{i}
$$

where $\mathbf{h}_{i}=\left[h_{i, 0}, h_{i, 1}, \cdots, h_{i, L-1}\right]^{T}$ denotes the channel impulse response (CIR) of length $L$ applied to the $i$ th TDSOFDM symbol,

$$
\boldsymbol{\Psi}_{i}=\left[\begin{array}{ccccc}
c_{i, 0} & x_{i-1, N-1} & x_{i-1, N-2} & \cdots & x_{i-1, N-L+1} \\
c_{i, 1} & c_{i, 0} & x_{i-1, N-1} & \cdots & x_{i-1, N-L+2} \\
c_{i, 2} & c_{i, 1} & c_{i, 0} & \cdots & x_{i-1, N-L+3} \\
\vdots & \vdots & \vdots & \ddots & \vdots \\
c_{i, L-1} & c_{i, L-2} & c_{i, L-3} & \cdots & c_{i, 0} \\
c_{i, L} & c_{i, L-1} & c_{i, L-2} & \cdots & c_{i, 1} \\
\vdots & \vdots & \vdots & \ddots & \vdots \\
c_{i, M-1} & c_{i, M-2} & c_{i, M-3} & \cdots & c_{i, M-L}
\end{array}\right],
$$

and $\mathbf{v}_{i}$ is the additive white Gaussian noise (AWGN) vector subject to the distribution of $\mathcal{C N}\left(\mathbf{0}, \mathbf{I}_{M} \sigma^{2}\right)$. It is clear that $\mathbf{d}_{i}$ will be contaminated by the last $L-1$ samples of the previous OFDM data block $\mathbf{x}_{i-1}$.

As illustrated in Fig. 3 (a), the iterative CIR estimation has to be implemented using the contaminated PN sequence, whereby a reliable result is difficult to achieve. The DPNOFDM solution in Fig. 3 (b) adopts two repeated PN sequences as the guard interval, so that the second PN sequence is not contaminated by the IBI from the preceding OFDM data block and can be used to realize accurate channel estimation, at the cost of an obviously reduced spectrum efficiency. In contrast, we propose a TDS-OFDM transmission solution based on simultaneous multi-channel reconstruction via structured CS, as shown in Fig. 3 (c). This solution has the following three key components.

First, the sparsity nature of wireless channels will be exploited. Numerous theoretical analyses and experimental results have confirmed that the wireless channels are sparse in nature, especially in broadband wireless communications [10]. More specifically, the CIR $\mathbf{h}_{i}=\left[h_{i, 0}, h_{i, 1}, \cdots, h_{i, L-1}\right]^{T}$ comprising of $S_{i}$ resolvable propagation paths is modeled as

$$
h_{i, n}=\sum_{l=0}^{S_{i}-1} \alpha_{i, l} \delta\left[n-\tau_{i, l}\right], 0 \leq n \leq L-1,
$$

where $\alpha_{i, l}$ is the gain of the lth path, $\tau_{i, l}$ is the delay of the $l$ th path normalized to the sampling period at the receiver, and 


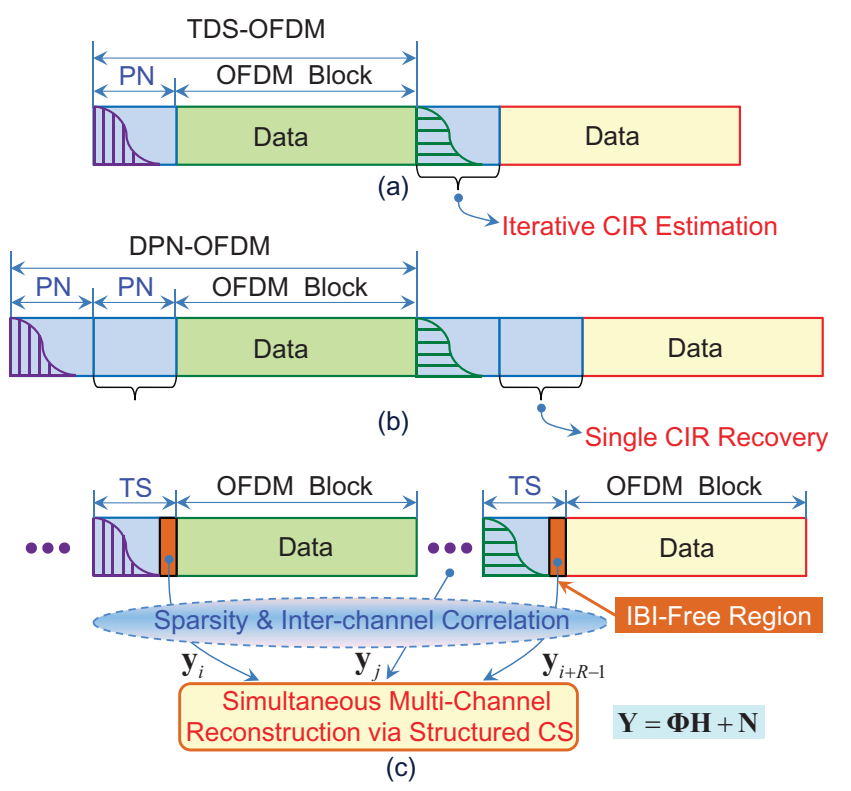

Fig. 3. The proposed TDS-OFDM based on simultaneous multi-channel reconstruction via structured CS, with the comparison with traditional solutions: (a) The conventional TDS-OFDM scheme; (b) The dual PN padding OFDM (DPN-OFDM) scheme; (c) The proposed solution.

the path delay set $D_{i}$ is defined as

$$
D_{i}=\left\{\tau_{i, 0}, \tau_{i, 1}, \cdots, \tau_{i, S_{i}-1}\right\} .
$$

The sparsity nature means that $S_{i} \ll L$.

Second, we will also exploit the inter-channel correlation caused by the fact that the path delays vary much slower than the path gains [11]. Even if the path gains are varying significantly from on symbol to the next symbol, the path delays during several successive TDS-OFDM symbols typically remain unchanged. This is caused by the fact that the coherence time of the fast time-varying path gains is inversely proportionally to the system's working carrier frequency, while the path delay variation is inversely proportionally to the signal bandwidth [11]. More specifically, the CIR column vectors for $R$ consecutive TDS-OFDM symbols can be assumed to share the same sparsity pattern [9], i.e.,

$$
\left\{\begin{array}{c}
S_{i}=S_{i+1}=\cdots=S_{i+R-1}=S, \\
D_{i}=D_{i+1}=\cdots=D_{i+R-1}=D, \\
\tau_{i, l}=\tau_{i+1, l}=\cdots=\tau_{i+R-1, l}=\tau_{l},
\end{array}\right.
$$

where $0 \leq l \leq S-1$. We define

$$
\mathbf{H}=\left[\mathbf{h}_{i}, \mathbf{h}_{i+1}, \cdots, \mathbf{h}_{i+R-1}\right],
$$

which is said to be jointly $S$-sparse, i.e., $\mathbf{H}$ has $S$ nonzero rows with indices $D$ in (5).

Third, we will further exploit the small-size IBI free region within the received PN sequence to realize simultaneous multichannel reconstruction. Since the guard interval length is such that the worst-case channel length is as large as the guard interval length, the actual channel length is usually smaller or even much smaller than the guard interval length in most practical scenarios [12], and then there exists an IBIfree region $\mathbf{y}_{i}=\left[d_{i, L-1}, d_{i, L}, \cdots, d_{i, M-1}\right]^{T}$ of small size $G=M-L+1$ not affected by IBI:

$$
\mathbf{y}_{i}=\boldsymbol{\Phi}_{i} \mathbf{h}_{i}+\mathbf{n}_{i}
$$

where $\mathbf{n}_{i}$ is the AWGN with distribution $\mathcal{C N}\left(\mathbf{0}, \sigma^{2} \mathbf{I}_{G}\right)$, and

$$
\boldsymbol{\Phi}_{i}=\left[\begin{array}{ccccc}
c_{i, L-1} & c_{i, L-2} & c_{i, L-3} & \cdots & c_{i, 0} \\
c_{i, L} & c_{i, L-1} & c_{i, L-2} & \cdots & c_{i, 1} \\
\vdots & \vdots & \vdots & \vdots & \vdots \\
c_{i, M-1} & c_{i, M-2} & c_{i, M-3} & \cdots & c_{i, M-L}
\end{array}\right]_{G \times L}
$$

corresponds to the last $G$ rows of the matrix $\Psi_{i}$ in (2). Considering the IBI-free regions of $R$ consecutive TDS-OFDM symbols with the same PN sequence (i.e., $\mathbf{c}_{i}=\mathbf{c}_{i+1}=\cdots=\mathbf{c}$, which leads to $\boldsymbol{\Phi}_{i}=\boldsymbol{\Phi}_{i+1}=\cdots=\boldsymbol{\Phi}$ ), we have

$$
\mathbf{Y}=\left[\mathbf{y}_{i}, \mathbf{y}_{i+1}, \cdots, \mathbf{y}_{i+R-1}\right]_{G \times R}=\mathbf{\Phi H}+\mathbf{N},
$$

where $\mathbf{N}=\left[\mathbf{n}_{i}, \mathbf{n}_{i+1}, \cdots, \mathbf{n}_{i+R-1}\right]_{G \times R}$. The considered mathematical model of (9) complies with the newly developed theory of structured CS [9], which is an extension of the standard CS theory [13]. Thus, the jointly sparse multiple channels within $\mathbf{H}$ can be simultaneously reconstructed by solving the following nonlinear optimization problem [9]:

$$
\widehat{\mathbf{H}}=\arg \min _{\mathbf{H} \in \mathbf{C}^{L \times R}}\|\mathbf{H}\|_{p, q}, \quad \text { subject to } \mathbf{Y}=\mathbf{\Phi H}+\mathbf{N} \text {, }
$$

where the $l_{p, q}$ norm of the matrix $\mathbf{H}$ is defined as

$$
\|\mathbf{H}\|_{p, q}=\left(\sum_{i}\left\|r^{i}\right\|_{p}^{q}\right)^{\frac{1}{q}}
$$

with $r^{i}$ being the $i$ th row of $\mathbf{H}$. Typically an $l_{2,0}$ norm is used in the CS literature [9]. Note that (9) reduces to (7) when $R=1$, but the required number of observations for reliable signal recovery would increase from $\mathcal{O}(S)$ to $\mathcal{O}\left(S \log _{2}(L / S)\right)$ when $R>\log _{2}(L / S)$ [14].

By jointly exploiting the sparsity nature of the channel, the property of inter-channel correlation, and the theory of structured CS, the ideal of exploiting the small-size IBI-free region of the received PN sequence to realize simultaneous multichannel reconstruction without any interference cancellation becomes feasible, and the mutually conditional relationship between the time-domain channel estimation and frequencydomain data demodulation in conventional TDS-OFDM can be decoupled without reducing the spectrum efficiency.

\section{Simultaneous Multi-Channel Reconstruction BASED ON A-SOMP}

This section proposes a reliable yet low-complexity simultaneous multi-channel reconstruction scheme which combines the well-known signal reconstruction algorithm called SOMP with the specific technical features of TDS-OFDM. 


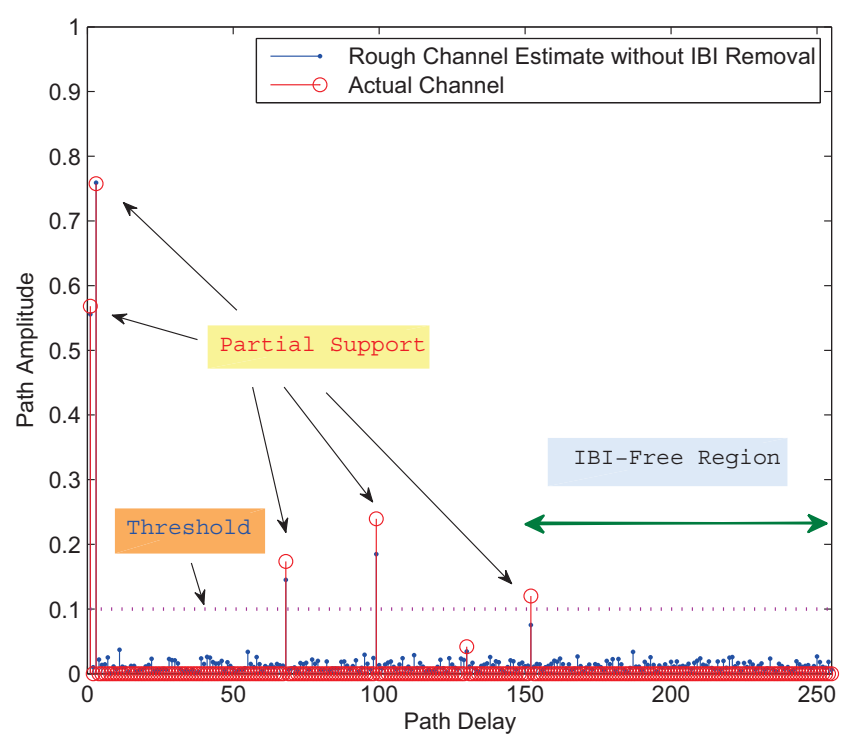

Fig. 4. Channel priori acquisition by using the contaminated PN sequence without IBI removal in the Vehicualr B channel with a SNR of $5 \mathrm{~dB}$.

\section{A. Correlation Based Channel Priori Acquisition}

Relying on the good auto-correlation properties of the PN sequence, without IBI removal, the received contaminated PN sequence is directly correlated with the local known PN sequence to generate a first rough channel estimate $\overline{\mathbf{h}}_{i}$ :

$$
\overline{\mathbf{h}}_{i}=\frac{1}{M} \mathbf{c}_{i} \otimes \mathbf{d}_{i}=\mathbf{h}_{i}+\mathbf{u}_{i},
$$

where $\mathbf{u}_{i}$ denotes the IBI effect caused by the previous OFDM data block as well as the AWGN. As illustrated in Fig. 4 where the Vehicular B channel [15] with a low signal-to-noise ratio (SNR) of $5 \mathrm{~dB}$ is simulated, the good autocorrelation properties of the PN sequence ensure that the main characteristics of the channel, especially the time delays of significant paths, can be preserved.

Based on the rough channel estimates $\overline{\mathbf{h}}_{i}$ during several consecutive TDS-OFDM symbols, the number of observation vectors $R$ needed to generate the observation matrix $\mathbf{Y}$ in (9) can be determined by checking the locations of the most significant taps within the rough estimates. Then, the path gains in $\overline{\mathbf{h}}_{i}$ are discarded, and the initial partial support of the jointly sparse channels could be approximated by

$$
D_{0}=\left\{l: \frac{1}{R} \sum_{j=i}^{i+R-1}\left|\bar{h}_{j . l}\right|^{2} \geq p_{t h}\right\}_{l=0}^{L-1},
$$

where $p_{t h}$ is the power threshold used to determine the main paths, which can be configured conservatively to ensure the correct information of the obtained partial support (e.g, $p_{t h}=$ 0.1 is used in Fig. 4).

The channel sparsity level $S$ is then approximated by

$$
S=S_{0}+a=\left\|D_{0}\right\|_{0}+a,
$$

where $S_{0}=\left\|D_{0}\right\|_{0}$ denotes the initial channel sparsity level according to the correlation-based rough channel estimate (12), and $a$ is a positive compensation number used to combat the

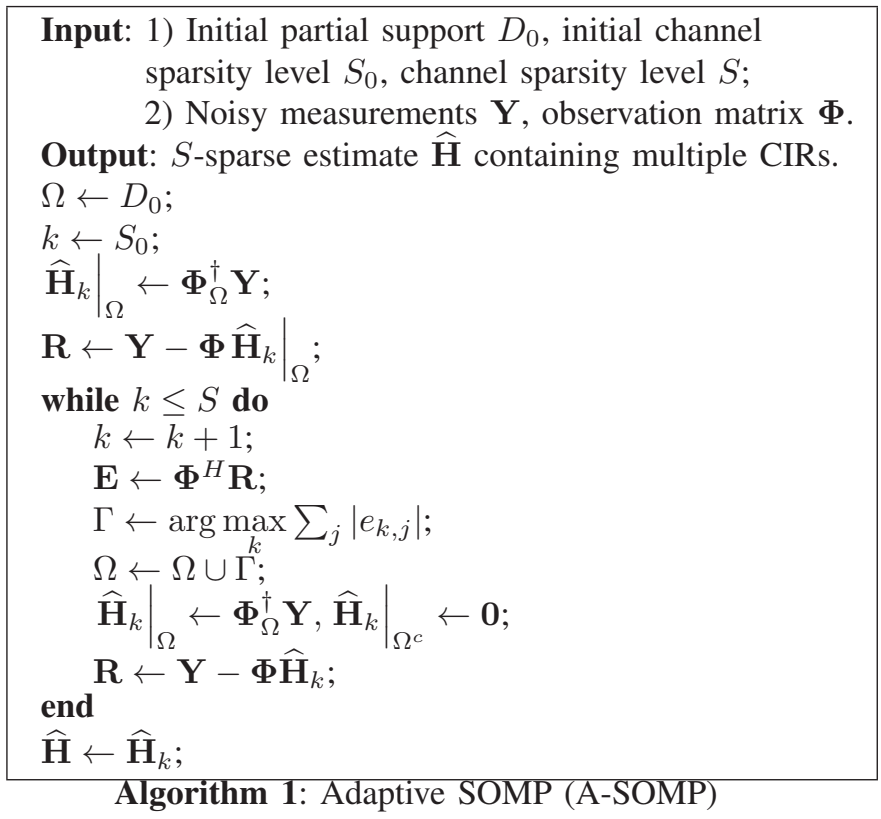

interference effect, since some low-power active paths may be treated as noise in (13).

Finally, the channel length $L$ can be estimated by

$$
\widehat{L}=\max \left\{D_{0}\right\}+b,
$$

where $b$ is the variable parameter used to define the IBIfree region comprising the last $G$ samples of the received PN sequence.

\section{B. A-SOMP Based Joint Sparsity Pattern Recovery}

Based on the basic principle of SOMP, we propose the A-SOMP algorithm, which is adaptive to variable channel conditions, and exploits the channel priori to reduce the complexity. The pseudocode of the proposed A-SOMP algorithm is summarized in Algorithm 1, which differs from SOMP [16] in the following three aspects:

1) Number of iterations. Since the partial support is already known, A-SOMP only executes $S-S_{0}$ iterations instead of $S$ iterations in SOMP.

2) Initialization. Since the initial support is known, the initial residual signal $\mathbf{R} \leftarrow \mathbf{Y}-\left.\mathbf{\Phi} \widehat{\mathbf{H}}_{k}\right|_{\Omega}$ is used to replace its counterpart $\mathbf{R} \leftarrow \mathbf{0}$ in SOMP, whereby $\boldsymbol{\Phi}_{\Omega}^{\dagger} \mathbf{Y}$ is the initial estimate of channel matrix $\mathbf{Y}$.

3) Adaptation. A-SOMP is adaptive to the channel sparsity level, the number of observation vectors, as well as the number of iterations.

After $\widehat{\mathbf{H}}$ has been obtained by the proposed A-SOMP algorithm, again the path gains within $\widehat{\mathbf{H}}$ are discarded, and the path delays of the nonzero taps are estimated by the support of $\widehat{\mathbf{H}}$ as follows

$$
\widehat{D}=\operatorname{supp}\{\widehat{\mathbf{H}}\} .
$$

Note that $S-S_{0}$ instead of $S$ iterations are carried out by the proposed A-SOMP algorithm, so it reduces the complexity 
of SOMP by a factor of $S_{0} / S$. For example, the complexity is reduced by about $66.67 \%$ if four out of six channel path delays have been obtained by the channel priori acquisition step.

\section{ML Based Path Gain Estimation}

After the path delays have been obtained, the signal model (7) is simplified to

$$
\mathbf{y}_{i}=\boldsymbol{\Phi}_{\widehat{D}} \mathbf{h}_{i S}+\mathbf{n}_{i},
$$

where $\mathbf{h}_{i S}$ is generated by restricting the vector $\mathbf{h}_{i}$ to its $S$ largest components, and $\boldsymbol{\Phi}_{\widehat{D}}$ is the submatrix comprising the $\widehat{D}$ columns of $\boldsymbol{\Phi}$. It is clear from (17) that there remains only $S$ instead of $L(S<G \ll L)$ unknown nonzero path gains in the CIR vector $\mathbf{h}_{i}$, which can be estimated by solving an over-determined equation under the ML criterion:

$$
\widehat{\mathbf{h}}_{i S}=\boldsymbol{\Phi}_{\widehat{D}}^{\dagger} \mathbf{y}_{i}=\left(\boldsymbol{\Phi}_{\widehat{D}}^{H} \boldsymbol{\Phi}_{\widehat{D}}\right)^{-1} \boldsymbol{\Phi}_{\widehat{D}}{ }^{H} \mathbf{y}_{i} .
$$

Finally, the path delay and path gain estimates form the complete CIR estimate as $\left.\widehat{\mathbf{h}}_{i}\right|_{\widehat{D}}=\widehat{\mathbf{h}}_{i S}$.

Similar operations (18) could be carried out to obtain the estimates of the remaining $R-1$ CIR vectors to finally accomplish the simultaneous multi-channel reconstruction.

For theoretical performance analysis purpose, we have derived the CRLB of the proposed reconstruction scheme as

$$
\mathrm{CRLB}=\mathrm{E}\left\{\left\|\widehat{\mathbf{h}}_{S}-\mathbf{h}_{S}\right\|_{2}\right\}=\frac{S \sigma^{2}}{G},
$$

which is smaller than the noise level $\sigma^{2}$ as $S<G$. Note that the detailed derivation is omitted here due to the page limitation.

\section{Simulation Results and Discussion}

Simulations are carried out to investigate the performance of the proposed 256QAM-supporting TDS-OFDM scheme. The simulation setup is configured according to typical wireless broadcasting systems [5]. The signal bandwidth is $7.56 \mathrm{MHz}$ located at the central radio frequency of $770 \mathrm{MHz}$. The FFT size $N=4096$ and the guard interval length $M=256$ are adopted. The modulation scheme uses 256QAM, and a lowdensity parity-check (LDPC) code with block length 64, 8000 bits and code rate 0.6 as specified in [7] is considered. The six-tap Vehicular B channel model [15] with the maximum delay spread of $20 \mu \mathrm{s}$ is adopted.

Fig. 5 shows the MSE performance comparison between the proposed simultaneous multi-channel reconstruction method based on A-SOMP and its counterparts in conventional TDSOFDM, DPN-OFDM, and CP-OFDM, for the long-delay Vehicular B channel. To ensure the channel estimation performance when the SNR is low, the last $G=30$ samples of the IBI-free region are selected for the joint CIR reconstruction. It is clear that the proposed scheme outperforms the conventional systems by more than $5 \mathrm{~dB}$ when the target MSE of $10^{-2}$ is considered. Moreover, the actual MSE performance approaches the theoretical CRLB (19) when the SNR becomes

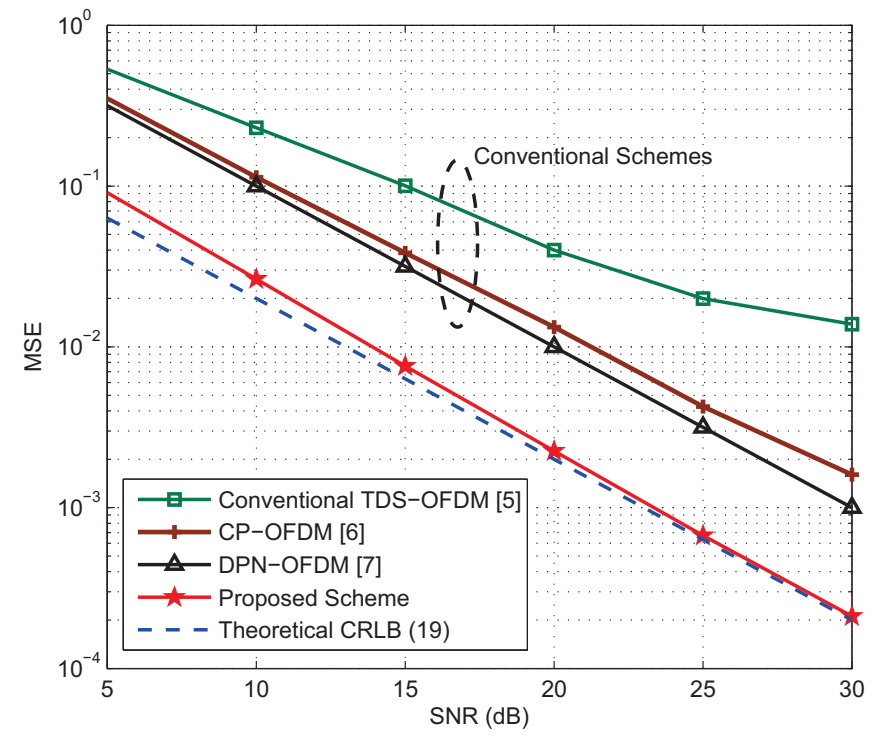

Fig. 5. Channel estimation performance comparison in the Vehicular B multipath channel with large delay spread.

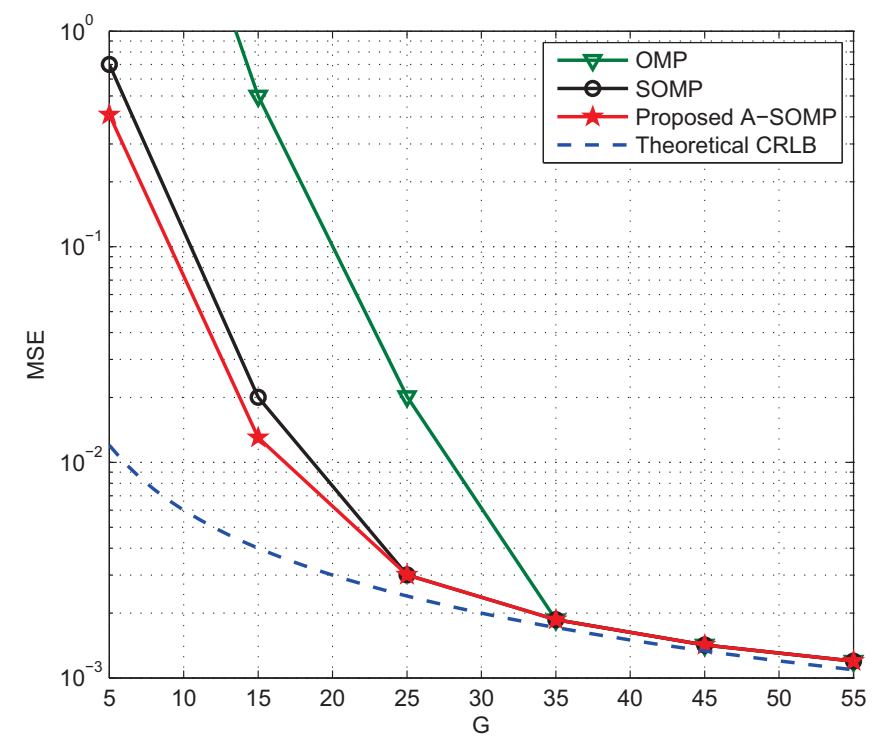

Fig. 6. Signal reconstruction performance comparison in the Vehicular B channel.

high. The accurate channel estimation is mainly contributed by the fact that the sparsity as well as the inter-channel correlation channel properties are fully exploited by the proposed ASOMP algorithm.

Fig. 6 presents the signal reconstruction performance comparison between the proposed A-SOMP algorithm and the traditional SOMP scheme when a varying number of measurements is utilized. The classical OMP algorithm for standard CS is also considered for comparison. Compared with OMP, both SOMP and A-SOMP require fewer observations to achieve the MSE of $10^{-2}$, since several observation vectors are utilized by SOMP and A-SOMP while only one vector is used by OMP. Meanwhile, A-SOMP performs slightly better than SOMP because the channel prior has been used. Note that the channel 


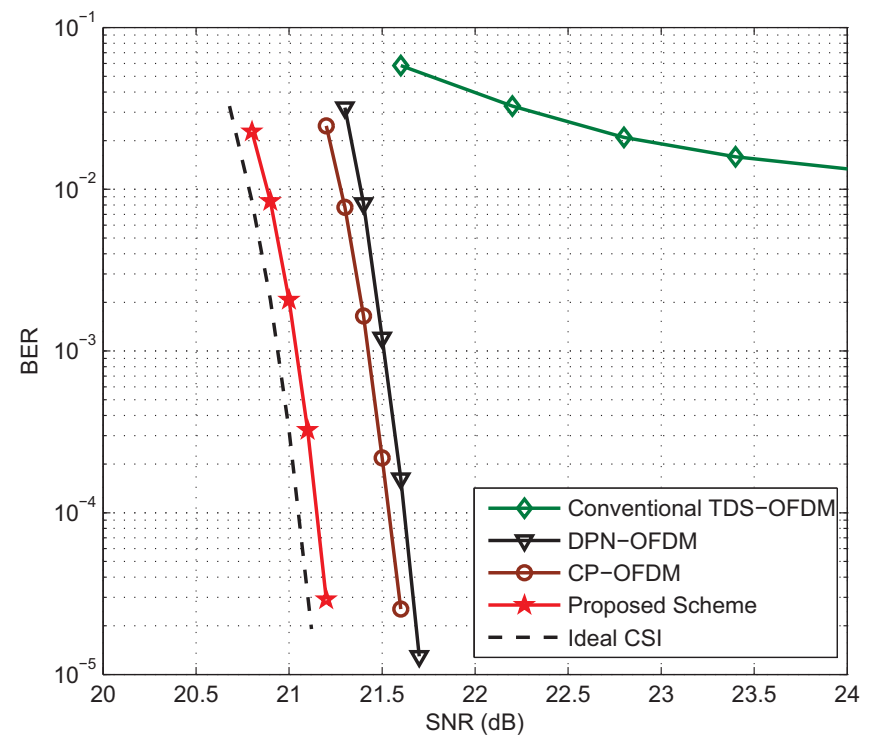

Fig. 7. Coded BER performance comparison when 256QAM is adopted in the Vehicular B channel.

prior is mainly used to reduce the complexity of SOMP as discussed in Section III-B. Moreover, it is clear that the signal reconstruction quality approaches the theoretical CRLB when the number of observations becomes large.

Fig. 7 compares the LDPC coded BER performance when 256QAM is adopted in the Vehicular B channel. The BER performance with the ideal channel state information (CSI) is also included as the benchmark for comparison. We observe that the conventional TDS-OFDM system can not support 256QAM because the residual interferences can not be removed well, while the proposed TDS-OFDM scheme can support 256QAM reliably, which leads to the improved spectrum efficiency of about 30\%. Moreover, owing to the decoupling of the time-domain channel estimation and frequency-domain data detection, as well as the improved channel estimation accuracy, the proposed scheme also has superior BER performance to DPN-OFDM and CP-OFDM with an SNR gain of about $0.5 \mathrm{~dB}$ and $0.4 \mathrm{~dB}$ at a BER of $1 \times 10^{-4}$, respectively. In addition, the actual BER curve is only about $0.1 \mathrm{~dB}$ away from the ideal CSI case, which indicates the excellent BER performance of the proposed scheme.

\section{CONCLUSIONS}

In this paper, we have presented a TDS-OFDM scheme with an improved spectrum efficiency of about $30 \%$ compared with the conventional system. The sparsity nature and interchannel correlation of wireless channels are jointly exploited, and multiple IBI-free regions of very small size within consecutive received TDS-OFDM symbols are utilized to realize a simultaneous multi-channel reconstruction with high accuracy under the framework of structured compressive sensing. In this way, not only an obviously improved channel reconstruction accuracy can be achieved, but also the mutually conditional time-domain channel estimation and frequency-domain data detection in conventional TDS-OFDM can be decoupled with- out the use of iterative interference cancellation. It has been shown that the proposed scheme can support 256QAM with a LDPC coded BER performance close to the ideal CSI case. Finally, it is noted that although the initial motivation of this paper is to enable TDS-OFDM to support 256QAM for higher spectrum efficiency, the proposed methods can complement TDS-OFDM as well as the so-called unique word single carrier (UW-SC) systems in any context (e.g., QPSK, 16QAM, 64QAM, etc) for BER performance enhancement.

\section{ACKNOWLEDGMENTS}

This work was supported by National Key Basic Research Program of China (Grant No. 2013CB329203), National Natural Science Foundation of China (Grant Nos. 61271266 and 61201185), and Tsinghua University-KU Leuven Bilateral Scientific Cooperation fund (Grant No. BIL11/21T). P. Tsiaflakis is a postdoctoral fellow funded by the Research Foundation-Flanders (FWO).

\section{REFERENCES}

[1] J. Ketonen, M. Juntti, and J. Cavallaro, "Performance-complexity comparison of receivers for a LTE MIMO-OFDM system," IEEE Trans. Signal Process., vol. 58, no. 6, pp. 3360-3372, Jun. 2010.

[2] Z. Wang and R. Stirling-Gallacher, "Frequency reuse scheme for cellular OFDM systems," Electron. Lett., vol. 38, no. 8, pp. 387-388, Apr. 2002.

[3] L. Dai, Z. Wang, and Z. Yang, "Time-frequency training OFDM with high spectral efficiency and reliable performance in high speed environments," IEEE J. Sel. Areas Commun., vol. 30, no. 4, pp. 695-707, May 2012.

[4] T. van Waterschoot, V. Le Nir, J. Duplicy, and M. Moonen, "Analytical expressions for the power spectral density of CP-OFDM and ZP-OFDM signals," IEEE Signal Process. Lett., vol. 17, no. 4, pp. 371-374, Apr. 2010.

[5] L. Dai, Z. Wang, and Z. Yang, "Next-generation digital television terrestrial broadcasting systems: Key technologies and research trends," IEEE Commun. Mag., vol. 50, no. 6, pp. 150-158, Jun. 2012.

[6] J. Wang, Z. Yang, C. Pan, and J. Song, "Iterative padding subtraction of the PN sequence for the TDS-OFDM over broadcast channels," IEEE Trans. Consum. Electron., vol. 51, no. 11, pp. 1148-1152, Nov. 2005.

[7] Digital Video Broadcasting (DVB); Frame Structure, Channel Coding and Modulation for a Second Generation Digital Terrestrial Television Broadcasting System (DVB-T2). ETSI Standard, EN 302 755, V1.3.1, Apr. 2012.

[8] J. Fu, J. Wang, J. Song, C. Pan, and Z. Yang, "A simplified equalization method for dual PN-sequence padding TDS-OFDM systems," IEEE Trans. Broadcast., vol. 54, no. 4, pp. 825-830, Dec. 2008.

[9] M. Duarte and Y. Eldar, "Structured compressed sensing: from theory to applications," IEEE Trans. Signal Process., vol. 59, no. 9, pp. 40534085, Sep. 2011.

[10] W. Bajwa, J. Haupt, A. Sayeed, and R. Nowak, "Compressed channel sensing: A new approach to estimating sparse multipath channels," Proc. IEEE, vol. 98, no. 6, pp. 1058-1076, Jun. 2010.

[11] I. Telatar and D. Tse, "Capacity and mutual information of wideband multipath fading channels," IEEE Trans. Inf. Theory, vol. 46, no. 4, pp. 1384-1400, Jul. 2000.

[12] G. Stuber, J. Barry, S. Mclaughlin, Y. Li, M. Ingram, and T. Pratt, "Broadband MIMO-OFDM wireless communications," Proc. IEEE, vol. 92, no. 2, pp. 271-294, Feb. 2004.

[13] D. Donoho, "Compressed sensing," IEEE Trans. Inf. Theory, vol. 52, no. 4, pp. 1289-1306, 2006.

[14] E. Van Den Berg and M. Friedlander, "Theoretical and empirical results for recovery from multiple measurements," IEEE Trans. Inf. Theory, vol. 56, no. 5, pp. 2516-2527, May 2010.

[15] Guideline for Evaluation of Radio Transmission Technology for IMT2000. Recommendation ITU-R M. 1225, 1997.

[16] Y. Eldar and H. Rauhut, "Average case analysis of multichannel sparse recovery using convex relaxation," IEEE Trans. Inf. Theory, vol. 56, no. 1, pp. 505-519, Jan. 2010. 